\title{
Hypnosis in 53 Children With Anxiety Disorders, Nocturnal Enuresis, or Insomnia
}

\author{
Carla Minosh ${ }^{\mathrm{a}, \mathrm{b}}$, Richard H. Schwartz ${ }^{\mathrm{b}, \mathrm{d}}$, Vahe Badalyan ${ }^{\mathrm{c}}$
}

\begin{abstract}
Background: We present results of a prospective long-term study of 53 children treated consecutively with hypnotherapy.

Study Design: Children were treated for anxiety, nocturnal enuresis, or insomnia. Long-term (mean of 25.4 months) follow-up was performed.

Results: Of the 20 children with anxiety disorder, 55\% had goodto-excellent long-term results, rating a 4 (good) to 5 (excellent) on a scale of 1 to 5 . Seventeen children were treated for nocturnal enuresis and $59 \%$ had good/excellent rating for long-term results. The 16 children with insomnia had long-term good/excellent long-term effectiveness of $37.5 \%$. No parent or child reported any adverse effect.
\end{abstract}

Conclusion: In this pilot study, hypnotherapy was performed by a professionally trained nurse practitioner. The use of hypnosis was safe and moderately helpful, particularly for anxiety disorder or nocturnal enuresis. Although results of this pilot study showed modest effectiveness, fair to poor re-visit compliance and poor compliance with at-home, self-hypnosis practice, reduced the ability to effectively rate our implementation of this treatment modality.

Keywords: Hypnosis; Hypnotherapy; Anxiety disorders; Nocturnal enuresis; Sleep disorder

Manuscript accepted for publication August 14, 2013

\footnotetext{
aAdvanced Pediatrics, Vienna, VA, USA

${ }^{b}$ Department of Pediatrics, Inova Children's Hospital, Falls Church, VA, USA

'Section of Gastroenterology, Hepatology, and Nutrition, Children's National Medical Center, Washington DC, USA

${ }^{\mathrm{d}}$ Corresponding author: Richard H. Schwartz, 100 East Street, SE, Suite 301, Vienna, VA 22180, USA. Email: rhs738@aol.com
}

doi: http://dx.doi.org/10.4021/ijcp104w

\section{Introduction}

Hypnosis is a form of mind and body practice, used as part of an integrative medicine approach. Integrative medicine is defined by the National Institutes of Health, National Center for Complementary and Alternative Medicine as the combination of non-mainstream modalities along with traditional medicine [1]. The American Psychological Association recognizes hypnosis as an effective therapeutic technique, useful for many conditions and disorders. They state that "hypnosis is a procedure during which a health professional or researcher suggests while treating someone that he or she experience changes in sensations, perceptions, thoughts, or behavior" [2].

While there are many definitions of hypnosis, the American Society of Clinical Hypnosis best defines it as "a state of inner absorption, concentration and focused attention". It is like using a magnifying glass to focus the rays of the sun and make them more powerful. Similarly, when our minds are concentrated and focused, we are able to use our minds more powerfully. Because hypnosis allows people to use more of their potential, learning self-hypnosis is the ultimate act of self-control [3]. While rapport is arguably the most important factor in the effectiveness of hypnosis, the patient's response sets are also tied to efficacy: their prior experiences, learning, and expectations. In addition, the hypnotherapist's ability to appropriately tie suggestions to feedback of observed responses has an effect on patient confidence in the hypnosis methods. Patient consent is essential to the process, as is their commitment to change and their resolve to work on their skills between sessions [4]. In-office hypnotherapy was performed by a nurse practitioner trained through basic and advanced courses through the American Society of Clinical Hypnosis, Education and Research Foundation. Table 1 reviews the components of the hypnotherapy used in this study.

Many articles in the medical literature suggest that hypnosis may be an effective method to help some children who manifest a variety of disorders [5-11]. These include symptom reduction for mild-to-moderate acute or chronic intermittent anxiety states, including phobias and compulsions 
Table 1. Medical Hypnosis Procedure

1. Therapist introduces self to child and parent

2. Assessment of the problem

3. Assessment of motivation and verbal agreement to attend several follow-up sessions and to practice selfhypnosis techniques at home.

4. Explanation and performance of the induction and relaxation phases using deep breathing techniques and visual imagery.

5. Deepening phase to facilitate hypnotic suggestion.

6. Gradual lightening phase until full alertness.

9. Post-hypnotic review of the session's goals.

10. Re-enforcement of goals by telephone or e-mail.

11. Follow-up office visits and repeat of steps 4-9.

12. Long-term follow-up to learn what works or did not work with that child and others with similar presenting problems, done as part of our own internal quality assessment.

$[7,12,13]$, sleep disorders, including nocturnal enuresis and delay of nocturnal sleep onset [13-19] acute or chronic pain reduction, including anticipatory pain reduction during painful procedures such as insertion of indwelling catheters [6], and chronic non-progressive headaches [20,21]. A literature search from PubMed and the Cochrane Databases revealed 60 publications, mostly case reports based on 2 to 60 cases, addressing the use of hypnotherapy in various child psychiatric conditions [22]. A meta-analysis of hypnosis treatment of nocturnal enuresis by Cochrane Systems Review concluded that "there was weak evidence to support the use of hypnosis [for this condition]" [23].

Advantages of medical hypnotherapy by a skilled provider include reduction or avoidance of pharmaceuticals, very small number of reported adverse effects, and an empa- thetic therapeutic modality.

During hypnotherapy sessions with children, the parent may remain in the room as passive observer, and is instructed on how to provide gentle reminders for the child to practice at home in a way that would minimize resistance, and to inquire about how often the child was practicing in a way that did not convey a judgmental opinion regarding the response.

There are many investigational studies of usually small numbers of children undergoing hypnotherapy of a long list of medical problems. Many publications on the subject only contain anecdotal data suggesting effectiveness of the procedure. Evidence-based effectiveness for the majority of integrative therapeutic modalities, including hypnosis, is often limited by poor documentation of study design or results. Control groups are usually lacking in published studies on

Table 2. Effectiveness of Hypnosis for Anxiety Disorders

\begin{tabular}{lllll}
\hline & Initial & \% & Long term & $\%$ \\
\hline 1: Poor & 6 & 30 & 6 & 30 \\
2: Fair & 3 & 15 & 3 & 15 \\
3: Good & 7 & 35 & 7 & 35 \\
4: Excellent & 4 & 20 & 4 & 20 \\
Grand total & 20 & 100 & 20 & 100 \\
\hline
\end{tabular}


Table 3. Self-Hypnosis Habits of Children Receiving Hypnotherapy for Anxiety Disorders

\begin{tabular}{lll}
\hline Row labels & Number & \% \\
\hline 1: Daily & 2 & 10 \\
2: A few times a week & 4 & 20 \\
3: A few times a month & 2 & 10 \\
4: Never & 12 & 60 \\
Grand total & 20 & 100 \\
\hline
\end{tabular}

the subject, including the present study. Although nocturnal enuresis may resolve spontaneously over time, the pediatric patients in this study who were treated for anxiety and insomnia did not have short-term situational anxiety and insomnia, but rather long-term and complex anxiety and insomnia issues, which are unlikely to spontaneously resolve. Books and reviews on the subject state that there is some promising evidence for effectiveness of hypnotherapy for a variety of disorders [24]

\section{Results}

In this article, we present methods and results of a prospective follow-up pilot study of 53 children divided into three groups (anxiety disorders, nocturnal enuresis, and delay of sleep onset disorder), treated with hypnosis. Hypnotherapy was performed by the senior author, a nurse practitioner newly trained in clinical hypnosis. After a full medical eval- uation and discussion of treatment options, and evaluation of the child's motivation to change as well as any barriers or secondary gain which could prevent change, hypnosis was offered to the families and chosen as a single modality, or in conjunction with other offered interventional therapies, such as an evaluation of sleep hygiene with recommendations for changes for insomnia, initiation of cognitive-behavioral therapy for anxiety, or limiting fluids before bedtime for nocturnal enuresis.

\section{Study population and study site}

The children in the study derive from a private pediatric practice in northern Virginia. Between 2009 and 2012, the study period, 5,270 children between 6 and 16 years of age were registered as patients. Of these, 69 children experienced at least one hypnotherapy session at our office.

There was only one licensed, certified hypnotherapist in our office during the study and she was the sole hypnothera-

Table 4. Effectiveness of Hypnosis for Nocturnal Enuresis

\begin{tabular}{lllll}
\hline Effectiveness & Initial & $\mathbf{\%}$ & Long-term & \% \\
\hline 1: Poor & 2 & 12 & 6 & 35 \\
2: Fair & 4 & 23 & 1 & 6 \\
3: Good & 5 & 29 & 7 & 41 \\
4: Excellent & 6 & 35 & 3 & 18 \\
(blank) & 17 & & 0 & 0 \\
Grand total & 99 & 17 & 100 \\
\hline
\end{tabular}


Table 5. Self-Hypnosis Practice at Home of Children Receiving Hypnotherapy for Nocturnal Enuresis

\begin{tabular}{lll}
\hline 1: Daily & 1 & 6 \\
2: A few times a week & 2 & 12 \\
3: A few times a month & 3 & 18 \\
4: Never & 11 & 64 \\
Grand total & 17 & 100 \\
\hline
\end{tabular}

pist and is lead author of this study. Another trained member of our medical staff made the telephone calls for short- and long-term effectiveness of hypnosis. A third person, the third author, calculated the outcome measurements for each of the three groups of patients studied.

Parents of pre-adolescents were requested to remain in the therapy room for all hypnotherapy sessions. Adolescents were given the option of hypnosis with or without the accompanying parent remaining in the room. Table 1 lists the steps in the initial office visit for hypnotherapy. At least annually, repeated attempts to contact one parent from each of our study children were made by a trained research assistant. At each follow-up telephone contact, parents were asked a set of questions inquiring, among other issues, about their assessment of the effectiveness of hypnosis for the presenting problem and observance of any hypnosis-related adverse effects. Children and adolescents were asked for their opinion as to the effectiveness of hypnosis for the stated problem if they returned for re-enforcement visits. We devised a 10-point subjective parent rating score with the following categories: poor $=1-2$; fair $=3-4$; good $=5-6$; excellent $=9-10$. Our scoring system was neither validated nor pilot-tested. Data were also collected about frequency of self-hypnosis at home, using techniques learned at the office hypnosis sessions.

This study is exempt from IRB review according to Category 4: "Research involving the collection or study of existing data, documents, records, pathological specimens, or diagnostic specimens, if these sources are publicly available or if the information is recorded by the investigator in such a manner that subjects cannot be identified, directly or through identifiers linked to the subjects."

This study was conceived after approximately 2 years of treating patients with hypnosis and using follow-up surveys to assess the effectiveness of the intervention as a means of improving quality in the practice. We used existing data from practice records. After the conception of the study, we continued to treat patients who presented for hypnotherapy, just as before, and continued our internal quality assessment as before, and used the records of the hypnosis sessions and the quality assessment follow-up questionnaires as the basis for the data in this study.

Initial outcomes following the hypnosis were assessed at irregular intervals, and depended upon many factors, including number of visits, when and whether the initial survey was returned, and whether the survey was completed during

Table 6. Effectiveness of hypnotherapy for delayed sleep onset disorder

\begin{tabular}{lllll}
\hline Effectiveness & Initial & \% & Long term & \% \\
\hline 0: not answered & 0 & 0 & 1 & 6 \\
1: Poor & 5 & 31 & 5 & 31 \\
2: Fair & 5 & 31 & 4 & 25 \\
3: Good & 3 & 19 & 2 & 25 \\
4: Excellent & 3 & 19 & 4 & 99 \\
Grand total & 16 & 100 & 16 & 25
\end{tabular}


a follow-up phone call if the initial survey was not returned. Since the outcomes were being assessed as part of our internal quality assurance, there were not strict protocols in place for follow-up. Outcomes for minor children were reported by the parent. As parents were instructed on how to remind a their child to practice in a way that minimized resistance, and to inquire about practice in a way that did not imply judgment about whether or not the child practiced, it is reasonable that the parents were aware of how much the child practiced.

Data examining the correlation between outcome of hypnosis and frequency of practice were not studied, as a specific plan for practice was not offered to these patients, but is being considered for future study, as we continue to improve the quality of our practice.

The data have been recorded in a way that the subjects cannot be identified. Parents and subjects all gave verbal consent for hypnosis and both were educated about hypnosis by the therapist. No advertisement was used to recruit patients. None of the authors has any financial conflict of interest. All payments were made to the private pediatric practice by parent co-pay at the time of service or by third party medical insurance.

During the 3.75-year duration of this pilot study, 69 consecutive evaluable children were seen for hypnotherapy for various problems. Of these, only 53 children are included in this report because the number of children who experienced hypnotherapy and learned self-hypnosis for specific other problems was too small for study analysis.

\section{Hypnotherapy for anxiety disorders}

The 20 children in the group treated for anxiety disorders consisted of 10 boys and 10 girls, ranging in age from 7 to 17 years. The mean age for this group was 10.5 years, and their median age was 11 years. Seventy percent of the children in this group had follow-up hypnosis sessions. Six children $(30 \%)$ had previously sought consultation, without parental assessment of effectiveness, from other professionals for anxiety disorders. Previous therapeutic attempts for 6 children included cognitive-behavioral therapy $(\mathrm{N}=3)$, and medication, with or without counseling $(\mathrm{N}=3)$. The mean follow-up period for the 20 children was 23.7 months. Table 2 gives specific data on initial and long-term effectiveness of hypnosis and Table 3 displays data on practicing selfhypnosis at home after the in-office hypnosis sessions. Of the 6 children who had unsuccessful previous therapy with cognitive-behavioral therapy or anxiolytic medication, 5 had an excellent or good initial response to hypnosis. Only six of $20(30 \%)$ children in this group practiced self-hypnosis at home on some regular basis.

In summary, the initial effectiveness response rating showed that the effectiveness of hypnosis was good-to-excellent in $55 \%$ and poor-to-fair in $45 \%$ of children with anxi- ety disorders. The long-term effectiveness rating score was $55 \%$ (good-to-excellent) and $45 \%$ (fair-to-poor).

\section{Nocturnal enuresis}

Seventeen children (10 boys and 7 girls) were treated with hypnosis for nocturnal enuresis. The mean age and median age of the group was 8.7 years.

Fourteen children ( $82 \%$ ) had experienced more than one hypnosis session: the mean number of follow-up sessions was 1.76. Table 4 displays data on initial and long-term parental rating of the effectiveness of hypnosis for the 17 children in this group and Table 5 displays the data on practicing self-hypnosis at home after the in-office hypnosis sessions.

Ten of the 17 children who had nocturnal enuresis $(59 \%)$ failed after active intervention with enuresis alarm or medication. Fourteen of the children in this group (82\%) had more than one hypnosis session. The mean number of follow-up sessions for the children for whom data are available was 1.5. Previous treatment for enuresis included: enuresis alarm, $\mathrm{N}=7$, medication, $\mathrm{N}=3$. Initial response to hypnosis for nocturnal enuresis was good-to-excellent in 11 children $(65 \%)$ and poor-to-fair in 6 children $(35 \%)$. The long-term response was good-to-excellent in 10 patients $(59 \%)$ and poor-to-fair in seven patients $(41 \%)$.

\section{Delay of onset of sleep disorder}

Sixteen children were treated by hypnosis for delayed sleep onset disorder. They included 11 girls and 5 boys with a mean age and median age of 10.4 years. Table 6 displays the parental rating of initial and long-term effectiveness of hypnosis for delay of onset of sleep disorder in this group. Of the 15 parent(s) who responded to our follow-up contacts, only $5(33 \%)$ children practiced self-hypnosis at home after the in-office hypnosis session. Previous measures to help induce sleep included melatonin (+/- music/sleep CD) in $10(62 \%)$ children. Eight children (50\%) had more than the initial hypnosis session (mean number of re enforcement sessions for the entire group $=0.5)$.

For delayed sleep onset, $6(37 \%)$ of parents rated the initial effectiveness of hypnosis as good-to-excellent and 6 $(37 \%)$ rated long-term effectiveness as good-to-excellent. Seven of the 16 children (44\%) in this group practiced hypnosis at home at least a few times per month. All children who did achieved a good-to-excellent result.

\section{Discussion}

There are very few published longitudinal studies on the use of hypnosis in the treatment of specific medical disorders in children $[5,23,24]$. One such long-term follow-up study of 505 children (who presented with many different reasons for 
requesting hypnotherapy), by Kohen et al, found that $50 \%$ of the children and adolescents achieved complete resolution of the presenting problem [5]. In that study, there was an inverse correlation between clinical success and number of re-enforcement visits for hypnotherapy. The Kohen et al study was published 29 years ago [5]. More recent follow-up studies on effectiveness of hypnosis for headaches in children and adolescents by Kohen [21] or Kohen and Zajac [20] have been published with longer follow-up periods. The authors of that study are acknowledged experts in hypnosis and represent the best possible outcomes. Moreover, these authors reported much better success with the percentage of patients who returned for hypnosis re-enforcement visits and for at home self-hypnosis practice.

Hypnosis techniques used by the lead author in the present study were less effective than techniques used by recognized experts, in large part because of our fair to poor rate of return visits and at home self-hypnosis practice [5, 20, 21]. Nevertheless, the initial success rate for the treatment of anxiety disorders and nocturnal enuresis can be called moderately successful. Pharmacological therapy with synthetic argenine desmopressin (DDAVP) has been shown to be $68 \%$ of 88 children after 6 months of therapy; only $10 \%$ of those who were considered successes remained dry after DDAVP had been discontinued for the next 6-month period [22].

Advantages of our study include the fact that we enrolled consecutive children who underwent hypnosis for one of three medical conditions, namely anxiety disorder, nocturnal enuresis, and delay onset sleep disorder. Our follow-up was up to 3.75 years, longer than any of the three studies by Kohen et al [5]. The hypnotherapist in our study was not a recognized expert and the hypnosis setting was a primary care pediatric practice, not a university center. Thus, the results of hypnosis by the lead author of the present study may be more representative of a pediatric primary care practitioner.

Our results suggest that integrative medicine studies can be done in a private office setting without major external funding. The present pilot study has several limitations. The number of enrollees was small, and, as such, our results must be considered as preliminary. We limited our study to children who underwent hypnosis for anxiety disorders, nocturnal enuresis, and delay of sleep onset. A major weakness of this study is the poor-to-fair participation in hypnosis re-enforcement office visits and fair-to-poor at-home selfhypnosis after the initial or re-enforcement sessions. Only $64 \%$ of the total study population attended more than the initial hypnosis session and only $34 \%$ practiced self-hypnosis in their homes. We learned from this follow-up study that we should have a signed commitment from parent and ageappropriate child to co-operate with re-enforcement sessions and home hypnosis practice sessions. The parent effectiveness rating scale that was used in this study was not validated nor pilot-tested prior to the study. Approximately $50 \%$ of the 53 children in the study groups had good-to-excellent par- ent assessment rating of effectiveness of hypnosis initially and during long-term follow-up. This is a lower success rate compared to data from Kohen et al, who are recognized experts in pediatric hypnosis [5].

These lapses may be remediated by written and verbal agreements between child and parent and child and therapist. At the initial visit for hypnotherapy, parents should be asked to attend at least two re-enforcement follow-up visits in the office, and the child should be asked to promise three times/week re-enforcement self-hypnosis for a minimum of 2 weeks. We anticipate that additional training and self-hypnosis practice sessions at home should improve our parents' effectiveness rating scores. Parents and subjects when available at the time of re-enforcement hypnosis sessions reported no adverse events from the hypnosis experience during or after the initial or re-enforcement sessions. Medical insurance was invoiced using the ICD-9 code for the final diagnosis (anxiety disorder, nocturnal enuresis, or delay sleep onset disorder).

How effective was hypnosis for the remaining 16 children treated with hypnotherapy but not included in these data? These included chronic intermittent headache or stomach ache $(\mathrm{N}=6)$, other source of pain $(\mathrm{N}=1)$, simple tic disorder $(\mathrm{N}=4)$, and miscellaneous complaints $(\mathrm{N}=5)$. Calculation of the outcome of these children revealed that $12 / 16(75 \%)$ had good-to-excellent parent hypnosis initial effectiveness rating scale and 3 poor-to-fair, with 1 individual data point showing no response to that question. The parent hypnosis follow-up rating scale results were: $9 / 16(56 \%)$ good-to-excellent and 6/16 (37\%) poor-to-fair. Therefore, the initial and long-term effectiveness of hypnosis in the remainder of children treated by hypnotherapy were quite similar to those of the 53 children who had anxiety disorders, nocturnal enuresis, or delay in sleep onset disorder.

Analysis of results from the 5 oldest children (age 16 to 17 years) did not rate higher in the initial or long-term parent effectiveness rating than analysis of results from the 5 youngest children (age 5 to 6 years).

In conclusion, based on results of this small pilot study performed by a newly trained nurse practitioner, we can make no definitive generalizable claim for the effectiveness of hypnosis for children who have the three disorders studied. The total parental rating of good-to-excellent initial effectiveness of hypnosis in our study was $29 / 53$ (55\%), and total good-to-excellent long-term response was $27 / 53$ (51\%). Long-term follow-up studies of the effectiveness of hypnotherapy should be replicated and improved by other investigators.

\section{Acknowledgement}

We owe a big thank you to Janeth Coca-Vargas for her diligence in contacting parents of children in our study in order 
to fill in missing data on our EXCEL sheet.

\section{References}

1. National Institutes for Health, National Center for Complementary and Alternative Medicine (NCCAM). (Accessed September 9, 2013). Complementary, Alternative, or Integrative Health: What's In a Name? http:// nccam.nih.gov/health/whatiscam.

2. American Psychological Association. (Accessed September 9, 2013). Hypnosis Today - Looking Beyond the Media Portrayal. http://www.apa.org/topics/hypnosis/ media.aspx.

3. American Society of Clinical Hypnosis. (Accessed September 9, 2013). Definition of Hypnosis. http://www. asch.net/Public/GeneralInfoonHypnosis/DefinitionofHypnosis/tabid/134/Default.aspx.

4. Lynn SJ, Hallquist MN. Toward a Scientifically Based Understanding of Milton H. Erickson's Strategies and Tactics: Hypnosis, Response Sets And Common Factors in Psychotherapy. Contemporary Hypnosis. 2004;21:6378.

5. Kohen DP, Olness KN, Colwell SO, Heimel A. The use of relaxation-mental imagery (self-hypnosis) in the management of 505 pediatric behavioral encounters. J Dev Behav Pediatr. 1984;5(1):21-25.

6. Eccleston C, Palermo TM, de CWAC, Lewandowski A, Morley S, Fisher E, Law E. Psychological therapies for the management of chronic and recurrent pain in children and adolescents. Cochrane Database Syst Rev. 2012;12:CD003968.

7. Kaiser P. Childhood anxiety, worry, and fear: individualizing hypnosis goals and suggestions for self-regulation. Am J Clin Hypn. 2011;54(1):16-31.

8. Waxman D. The development of contemporary hypnotherapy. Br J Hosp Med. 1994;51(4):192-193.

9. Olness KN. Hypnotherapy in children. New approach to solving common pediatric problems. Postgrad Med. 1986;79(4):95-100, 105.

10. Olness K, Gardner GG. Some guidelines for uses of hypnotherapy in pediatrics. Pediatrics. 1978;62(2):228-
233.

11. Saadat H, Kain ZN. Hypnosis as a therapeutic tool in pediatrics. Pediatrics. 2007;120(1):179-181.

12. Hobbie C. Relaxation techniques for children and young people. J Pediatr Health Care. 1989;3(2):83-87.

13. Porter J. Guided fantasy as a treatment for childhood insomnia. Aust N Z J Psychiatry. 1975;9(3):169-172.

14. Anbar RD, Slothower MP. Hypnosis for treatment of insomnia in school-age children: a retrospective chart review. BMC Pediatr. 2006;6:23.

15. Anderson JA, Dalton ER, Basker MA. Insomnia and hypnotherapy. J R Soc Med. 1979;72(10):734-739.

16. Graci GM, Hardie JC. Evidenced-based hypnotherapy for the management of sleep disorders. Int J Clin Exp Hypn. 2007;55(3):288-302.

17. Seabrook JA, Gorodzinsky F, Freedman S. Treatment of primary nocturnal enuresis: A randomized clinical trial comparing hypnotherapy and alarm therapy. Paediatr Child Health. 2005;10(10):609-610.

18. Huang T, Shu X, Huang YS, Cheuk DK. Complementary and miscellaneous interventions for nocturnal enuresis in children. Cochrane Database Syst Rev. 2011;12:CD005230.

19. Monda JM, Husmann DA. Primary nocturnal enuresis: a comparison among observation, imipramine, desmopressin acetate and bed-wetting alarm systems. J Urol. 1995;154(2 Pt 2):745-748.

20. Kohen DP, Zajac R. Self-hypnosis training for headaches in children and adolescents. J Pediatr. 2007;150(6):635639.

21. Kohen DP. Long-term follow-up of self-hypnosis training for recurrent headaches: what the children say. Int J Clin Exp Hypn. 2010;58(4):417-432.

22. Huynh ME, Vandvik IH, Diseth TH. Hypnotherapy in child psychiatry: the state of the art. Clin Child Psychol Psychiatry. 2008;13(3):377-393.

23. Working Party of the British Psychological Society. The Nature of Hypnosis. 2001; British Psychological Society, Leicester LEI 7DR, England.

24. Daniel P. Kohen, Karen Olness. Hypnosis and hypnotherapy with children. 4th ed. Routledge, 4th ed. 2011, New York. 